\title{
COMMENT
}

\section{May 2020 ECI biocommentary}

\author{
Eniko Szakmar ${ }^{1}$
}

Pediatric Research (2020) 87:977; https://doi.org/10.1038/s41390-020-0811-z

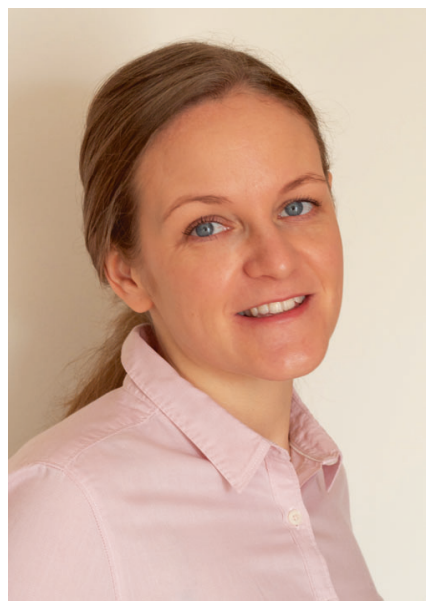

After graduating as a medical doctor with summa cum laude in 2015 from Semmelweis University, Budapest, Hungary I went on to purse a PhD degree in clinical medicine under the supervision of Dr. Agnes Jermendy. Currently, I am a PhD candidate and a Pediatric Resident at the 1st Department of Pediatrics, Semmelweis University.

Since medical school I have a special interest in therapy optimization of children who were born with neonatal encephalopathy (NE), which affects 1-3 infants per 1000 full-term live births in developed countries and related to one-quarter of the 4 million annual neonatal deaths worldwide. I have been moved by the countless cases that I saw in the past 6 years during my clinical research. The magnitude of the problem is clear from both a personal and an economic perspective and my ultimate goal is to find early intervention procedures, available already in the neonatal period, rather than let these children face belated rehabilitation efforts in the adulthood.
Adjunctive pharmacological agents have been heavily investigated over the last decades; however, the optimization of the intensive care including monitoring techniques may be a reasonable approach to improve long-term outcomes in infants with NE. The HENRIC trial was an investigator initiated clinical trial with the aim to test the safety and feasibility of adding carbon dioxide to the inhaled gas mixture in low concentration to correct hypocapnia in infants underwent therapeutic hypothermia. These kinds of studies are rare in neonatology due to their complexity; however, the findings of such trials cloud have a great impact on the clinical care of infants in the future.

During my PhD studies I have been really fortunate to work with very knowledgeable and highly dedicated clinicians and researchers, many of whom are coauthors on the paper of the HENRIC trial. In addition, I also had the opportunity to spend a considerable time abroad that broadened my horizons. A short fellowship at Cambridge University Hospital enabled me to learn about the advanced respiratory management in neonatal intensive care. I also spent one year at the Department of Pediatric Newborn Medicine in Brigham and Women's Hospital, Boston as a postdoctoral research fellow. I have been honored to work under the mentorship of Dr. Mohamed El-Dib focusing on the neurocritical care of newborns.

I strongly believe that a medical doctor should combine the regular clinical work with research and teaching activities to provide the best care for patients and to facilitate scientific and therapeutic advances.

\section{ADDITIONAL INFORMATION}

Competing interests: The author declares no competing interests.

Publisher's note Springer Nature remains neutral with regard to jurisdictional claims in published maps and institutional affiliations.

\footnotetext{
${ }^{1}$ 1st Department of Pediatrics, Semmelweis University, Budapest, Hungary
}

Correspondence: Eniko Szakmar (szakmar.eniko@med.semmelweis-univ.hu)

Received: 23 January 2020 Accepted: 1 February 2020

Published online: 15 February 2020 